\title{
A STATE OF ART ON CONTENT BASED IMAGE RETRIEVALSYSTEMS
}

\author{
V. Elizabeth Jesi, Dr. S. Govindarajan, M. Jayanthi
}

\begin{abstract}
The digital image data is quick expanding in capacity and heterogeneity. The customary information retrieval approaches are cannot fulfill the client's need, so there isneed to present a proficient framework for Content Based Image Retrieval(CBIR). The CBIR is an appealing wellspring of precise and quick retrieval. CBIR goes for discovering imagedatabases for explicit images that are like a given query image dependent on its features.In this paper the methodology of content based image retrieval are examined, investigated and thought about. Here, the different image substance, for example, colour, texture and shape features are mined by utilizing differentfeature extraction procedures, and furthermore extraordinary distance measures, Relevance Feedback $(R F)$ and indexing methods are used to improve the execution of the CBIR system.The existing exploration strategies are talked about with their benefits and negative marks, so the further research works can be focused more.
\end{abstract}

Index terms: Content Based Image Retrieval (CBIR), feature extraction, similarity measurement and Relevance Feedback (RF).

\section{INTRODUCTION}

Digital images play a significant task in regular daily existence. In numerous zones of science, commerce and government images are day by day obtained and utilized. Amid the previous decades have been watching a perpetual increment of image information, prompting large storehouses. The national geographic imagery archive of the United States (US) as of now has a size in the scope of PetaBytes (PB) and every day develops by a few TeraBytes (TB). The business Internet web crawler Yahoo professes to have ordered about 1.6 billion pictures. This fast development triggers the interest of subjective and quantitative image retrieval frameworks [1]-[3]. To date, different business frameworks and Internet web indexes features for keyword-based image retrieval.

Revised Manuscript Received on July 05, 2019.

V. Elizabeth Jesi, Asst. Prof., Dept of IT, SRM Institute of Science \& Technology.E-mail:jesi.v@ktr.srmuniv.ac.in

Dr.S. Govindarajan, Prof., Dept of EDP, SRM Institute of Science \& Technology.

M.Jayanthi, Asst. Prof., Dept of IT, SRM Institute of Science \& Technology.
As a rule, the outcomes are not agreeable because of the high difficulty of images that can only with significant effort be portrayed by words. In this manner, Content-Based Image Retrieval (CBIR) picked up in significance amid the previous decade.

Content Based Image Retrieval (CBIR) is a system in which for a given query picture, pertinent pictures will be recouped from an enormous picture database dependent on their substance closeness [4]. In CBIR, visual highlights, for example, colour and texture are removed to portray images. CBIR looks to maintain a strategic distance from the utilization of printed question inputs. Or maybe, it recovers pictures dependent on their visual similitude to a client provided query. The computer must be equipped for recovering pictures from a database with no human presumption on explicit space.

Initially, for each image in the image database, its visual contents are extracted - a set of distinguishing features is pre-computed via off-line feature extraction process [5]. The feature vector is then put away in an element metadata store. To extract images, the client presents a question model picture to the framework and the precedent picture is then changed over into an inner feature vector by means of on-line feature extraction process. The closeness among the feature vector of the client's query image and those element metadata things is determined and positioned amid likeness correlation arrange. Recovery is performed by applying an indexing plan which can be utilized to help quick recovery and to make the framework adaptable to vast picture databases. On the off chance that the recovery results because of the question are not completely agreeable, the client can give some positive or negative input to the framework and the adjusted inquiry can be resubmitted by means of the intelligent pertinence criticism. Such feedback-retrieval cycle can be rehashed until the client is happy with the recovery results.

\section{LITERATURE SURVEY}

Li et al (2002) planned a successful content based image recovery framework. This framework significantly incorporates two key segments: visual substance extraction and indexing, and inquiry engine [6]. The visual highlights ought to be

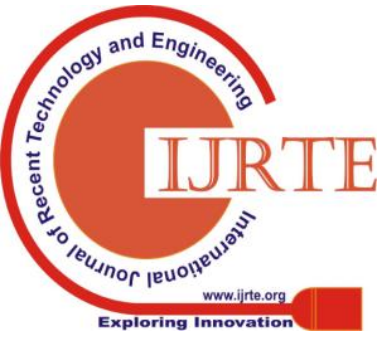


mined before pictures are recuperated. In this framework, the colour highlight is indicated by a 13-bincolour mark histogram which has been resolved. The spatial data is meant by class parameters which has been accomplished by concerning an unsupervised division mechanism merged with the wavelet plan to pictures. At long last to assess two images, the similarity between query and database must be resolved. In this work, L1-Distance, L2-Distance, and Quadratic Distance Metric based likeness estimation are performed dependent on the separated highlights. It accomplishes high recovering precision.

Huang et al (2010) designed a new CBIR framework based with respect to the colour minute and gabor texture element. In this work, the colour picture space is changed over into HSV shading space. So as to additionally diminish the element vector measurement, accelerate recovery use the centre moment which is to clarify histogram. In colour highlight extraction, colour moment of the Hue, Saturation and Value (HSV) segment pictures in the HSV are removed. The Gabor surface descriptors are used to remove the texture highlights. At last the image similarity is figured dependent on the separation estimation. The database and query image comparability is performed dependent on the Euclidean separation. Trial results demonstrate that the planned plan has predominant recovery precision than traditional plans utilizing color and texture highlights [7].

Chen and Zhao (2010) organized a RF for picture recovery. The RF is a fit arrangement for CBIR and it is similarly a sensible development to assemble the semantic gap among low-level visual segment and high level discernment. SVM-based RF plot is planned to propel the presentation of picture recuperation. In classifier setting up, a model growing framework is recognized to quality the degree of cheerful examples and unconstructive examples. After that a combination organize, the distinctive classifiers subject to versatile weighting is planned to pick the last question results. SVM-based RF plot is familiar with augmentation the execution of picture recuperation. In classifier setting up, an example expanding system is set up to adjust the extent of idealistic and unconstructive examples and a while later blend approach for various classifiers subject to adaptable weighting is obvious to pick the last inquiry results [8].

Quellec et al (2010) designed a novel wavelet advancement system for substance based image recovery in medicinal databases. In this work, without considered area express features the pictures are requested in a nonexclusive style. From the wavelet change a mark is developing for each picture. These picture marks depict the portion of wavelet coefficients in each sub band of the deterioration. Weights are also initiated among sub bands. The weight values are optimized. In order to compare two image signatures distance measurement is computed. While the doctor presents the inquiry increasingly comparable images are recovered. To extricate comparative images from a medicinal database, the marks and the separation assurance should be connected to the restorative clarification of pictures [9].

Bian et al (2010) designed a Biased Discriminant Euclidean Embedding (BDEE) system for image recovery. The BDEE accurately mold both the intra class geometry and interclass bias and not at all meet the under example trouble. In low-dimensional space, expanse among optimistic samples and unconstructive samples must be as higher as probable while distance among optimistic samples must be as minimum as possible. The confined geometry of optimistic samples should be conserved as greatly as possible by maintaining linear renovation coefficients acquired in. In this work, to get retrieved images assigning the query images together with optimistic samples, unconstructive samples, and the taken images are compare with the database. It achieves high accuracy [10].

Yuea et.al (2011) designed a color and texture fused features based content-based image retrieval. Initially, HSV color space is enumerated reasonably. The color space wants to be splitted into numerous tiny ranges to compute the color histogram. Every gap is observed as a bin. Thus, the color is computed. The color histogram can be determined through including pixels where the colors drop into every period. According to the co-occurrence matrix the texture information's are extracted.. The colour and texture features are combined by build weights of feature vectors. Finally based on the similarity the images are retrieved [11].

$\mathrm{Su}$ et al (2011) designed a productive RF for CBIR by extricating client route designs. For the most part the CBIR framework partitioned into two fundamental activities, namely offline knowledge detection and online image retrieval. For online procedure, when an inquiry picture is given to this framework, the plan initially decides the most related pictures without including any hunt system. At that point at long last returns a lot of the more comparable pictures. The main query conspire is known as starting input. By using the route designs, three search plans, with deference to Query Point Movement (QPM), Query Reweighting (QR), and Query Expansion (QEX), are joined to find the ideal pictures. In general, at each criticism, the results are gotten to the client and the associated perusing data is gathered in the log database. After gathering long haul clients' perusing behaviors, offline process for learning identification is 
actuated to execute route design mining and example ordering [12].

Murala et al (2012) structured another feature descriptor for CBIR. A presented new descriptor is called as Local Tetra Patterns (LTrPs). In this work, presented a novel picture indexing and recovery plot utilizing local tetra patterns (LTrPs) for substance based picture recovery. The basic Local Binary Pattern (LBP) and Local Ternary Pattern (LTP) code the relationship among the referenced pixel and its neighboring neighbors by figuring faint measurement refinement. The orchestrated arrangement encodes the relationship among the referenced pixel and its neighbors, as indicated by the bearings that are figured by utilizing the essential solicitation subordinate in vertical and flat requests. Moreover planned a nonexclusive plan to compute nthrequest $\mathrm{LTrP}$ by using (n-1) th-request level and vertical subordinates for accomplish better CBIR and look at the effectiveness of structured framework by blending it with the Gabor transform[13].

Liu and Yang (2013) designed Color Difference Histogram $(\mathrm{CDH})$ based CBIR approach. A presented descriptor blend the activity of introduction, orientation, color and color difference and gauge the spatial layout without the activity of any picture division plans. In this work, the RGB shading space is changed over into $\mathrm{L}^{*} \mathrm{a} * \mathrm{~b} *$ shading space. The $\mathrm{L}^{*} \mathrm{a} * \mathrm{~b} *$ shading space is a fundamentally decent brilliance for registering the distinction among hues, and the difference among two shading focuses can be determined as an Euclidean separation. At that point the edge introduction recognition and shading quantization is performed in $\mathrm{L}^{*} \mathrm{a} * \mathrm{~b} *$ colour spaces. In this designed algorithm, only edge orientations and color index values are chosen to compute the color difference histogram. The designed descriptor includes two special histograms categories, which are calculated in an equivalent way below the surroundings of colors and orientations. The $\mathrm{CDH}$ is utilized to define the image features for image retrieval [14].

Malik and Baharudin (2013) planned another CBIR utilizing factual quantized histogram texture highlights. In the initial stage, input RGB shading picture is changed into grayscale picture. The dark scale picture is partitioned into non covering squares. At that point apply Discrete Cosine Transformation (DCT) to all the non covering squares. Then the finish DCT changes, the DC coefficients in the each square and the essential three AC coefficients arebchosen in

zigzag arrangement. These coefficients are utilized to build histograms. At long last the comparability is estimated [15].

Bhute and meshram (2013) planned another content based image indexing and recovery framework. In this work, use color, texture and shape of inputs to help the recovery procedure. For better extraction, mine the color, texture and shape information of inputs routinely utilizing edge recognition which is extensively used in signal processing and image compression. In colour feature mining scheme, image histogram and colour correlogram are rapid recovery executes the antipole-tree scheme for indexing the images. Finally histogram Euclidean separation is performed to figure the likeness among database and query image [16].

Munje and kapgate (2014) designed a novel CBIR system. In CBIR scheme, the colour and texture information's are mined from query images. The color feature is mined by using color moment and texture details are mined by utilizing gabor filter and wavelet transform. The information's are computed for all images of query and database images. Totally 15 features for every image are determined, 6 color feature and residual are texture feature. Finally according to the similarity measure the significant images are extracted [17].

Fu et al (2016) present the Convolution Neural Network (CNN) based deep feature extraction methods. The CBIR framework uses the direct Support Vector Machine (SVM) to prepare a hyperplane which can isolate the comparative picture sets and different picture sets to an immense degree. The pair of features from input image and every investigation image in the image dataset is given as information (input). The test images at that point are reviewed by the separation among the pair features and the certified hyperplane. Tests demonstrate that the planned scheme can considerably increase the execution of CBIR for picture recovery undertakings [18].

Alsmadi (2017) designed an new similarity scheme for CBIR using memetic approach. In this work, color, shape and color texture informations are mined from query images. The shape features are used to extract the belongings of the shape of the pictures. The texture features are extracted by using GLCM which is robust image statistical analysis approach. Then the similarity is computed among the extracted feature and database feature by using memetic algorithm. Finally the performance of the work is analyzed [19]. 


\section{COMPARITIVE ANALYSIS}

\begin{tabular}{|c|c|c|c|}
\hline S.No & $\begin{array}{c}\text { Authors \& } \\
\text { Title }\end{array}$ & Merits & Demerits \\
\hline 1. & Ref : 6 & $\begin{array}{l}\text { High queue } \\
\text { processing speed }\end{array}$ & $\begin{array}{l}\text { Wavelet-based texture } \\
\text { feature is required to } \\
\text { improve the result }\end{array}$ \\
\hline 2. & Ref : 7 & $\begin{array}{l}\text { High accuracy } \\
\text { Fast retrieval } \\
\text { speed }\end{array}$ & $\begin{array}{l}\text { The designed method } \\
\text { does not allocate } \\
\text { various weight to } \\
\text { features based on the } \\
\text { image content } \\
\text { Instability }\end{array}$ \\
\hline 3. & Ref: 8 & Accentable & Need to improve the \\
\hline 4. & Ref : 9 & $\begin{array}{l}\text { High mean } \\
\text { precision value }\end{array}$ & $\begin{array}{l}\text { Better optimization } \\
\text { algorithm is required to } \\
\text { fine tune the parameter }\end{array}$ \\
\hline 5. & Ref : 10 & High accuracy & $\begin{array}{l}\text { The BDEE's semi- } \\
\text { supervised does not } \\
\text { considered the unlabelled } \\
\text { samples to recover the } \\
\text { relevance feedback } \\
\text { performance. }\end{array}$ \\
\hline 6. & Ref : 11 & $\begin{array}{l}\text { It achieves } \\
\text { better retrieval } \\
\text { results }\end{array}$ & $\begin{array}{l}\text { It does not include low- } \\
\text { level features such as shape } \\
\text { and spatial location } \\
\text { information for improve the } \\
\text { results. }\end{array}$ \\
\hline 7. & Ref : 12 & $\begin{array}{l}\text { More accurate } \\
\text { results }\end{array}$ & $\begin{array}{l}\text { It does not considered the } \\
\text { user's profile into NPRF to } \\
\text { enlarge the retrieval quality }\end{array}$ \\
\hline 8. & Ref : 13 & $\begin{array}{l}\text { High average } \\
\text { precision High } \\
\text { average recall }\end{array}$ & $\begin{array}{l}\text { Need to consider diagonal } \\
\text { pixels for derivative } \\
\text { calculations } \\
\text { improves the retrieved } \\
\text { results }\end{array}$ \\
\hline 9. & Ref : 14 & $\begin{array}{l}\text { It has superior } \\
\text { bias power of } \\
\text { color, texture, } \\
\text { shape information }\end{array}$ & $\begin{array}{l}\text { Need to improve the } \\
\text { retrieval accuracy }\end{array}$ \\
\hline 10. & Ref : 15 & $\begin{array}{l}\text { High precision } \\
\text { and F-Score }\end{array}$ & $\begin{array}{l}\text { It does not include various } \\
\text { features for improve the } \\
\text { retrieval system. }\end{array}$ \\
\hline 11. & Ref : 16 & High accuracy & $\begin{array}{l}\text { Include various similarity } \\
\text { measures to improve the } \\
\text { results }\end{array}$ \\
\hline 12. & Ref : 17 & High efficiency & $\begin{array}{l}\text { It does not possible for } \\
\text { more queries arrived at } \\
\text { same time }\end{array}$ \\
\hline 13. & Ref : 18 & $\begin{array}{l}\text { High retrieving } \\
\text { speed }\end{array}$ & $\begin{array}{l}\text { SVM does not possible for } \\
\text { large data set }\end{array}$ \\
\hline 14, & Ref : 19 & High precision & $\begin{array}{l}\text { Filtering techniques are } \\
\text { required to obtain more } \\
\text { precise results }\end{array}$ \\
\hline
\end{tabular}

\section{CONCLUSION}

In the current situation image retrieval plays a fundamental role. The image search and recovery from vast picture database is troublesome assignment. In recent years CBIR is increasing more consideration for investigator to tackle this issue. The CBIR isfamous methodology for recover an assortment of pictures from a lot of image database. The CBIR utilizes the image visual substance for instance colour, shape and texture to file and denote the images. For better CBIR framework, important to improve different recovery procedure, for example, Feature extraction, distance measurement technique and soon. In this paper, detailed on CBIR with feature extraction, similarity measurement and relevance feedback mechanisms and merits and demerits are investigated.

\section{REFERENCES}

[1] Hassekar P.P.; Sawant R.R., , "Experimental analysis of perceptual based texture features for image retrieval," in International Conference on Communication, Information \& Computing Technology (ICCICT), 2015.

[2] A. Smeulders, M. Worring, S. Santini, A. Gupta and R. Jain(2000), 'Content-based image retrieval at the end of the early years', IEEE Transactions on Pattern Analysis and Machine Intelligence 22 1349-1380.

[3] Yong-Hwan Lee, Sang-Burm Rhee, Bonam Kim, 'Content-based Image Retrieval Using Wavelet SpatialColor and Gabor Normalized Texture in Multi-resolution Database', Innovative Mobile and Internet Services in Ubiquitous Computing (IMIS), 2012 Sixth International Conference on , pp: $371-377,2012$.

[4] H.Alraqibah, M. M. Ben Ismail and O. Bchir, "Empirical Comparison of Visual Descriptors for Content based X-ray Image Retrieval", International Conference on Image and Signal Processing (ICISP'14), Cherbourg, June 2014.

[5] Ben Ismail M. M. and Bchir O., Content based Video Categorization using Relational Clustering with Local Scale Parameter, International Journal of Computer Science and Information Technology 8(1)(2016), 27-46.

[6]Li, X., Chen, S.C., Shyu, M.L. and Furht, B., 2002. An effective content-based visual image retrieval system.In Computer Software and Applications Conference, 2002.COMPSAC 2002.Proceedings. 26th Annual International (pp. 914-919). IEEE.

[7]. Huang, Z. C., Chan, P. P., Ng, W. W., \&Yeung, D. S. (2010, July). Content-based image retrieval using color moment and Gabor texture 
feature. In Machine Learning and Cybernetics (ICMLC), 2010 International Conference on (Vol. 2, pp. 719724).IEEE.

[8] Hengchen, zhichengzhao "an effective relevance feedback algorithm for image retrieval" 978-1-4244-68539/10/ 2010 IEEE.

[9]. Quellec, G., Lamard, M., Cazuguel, G., Cochener, B., \& Roux, C. (2010). Wavelet optimization for content-based image retrieval in medical databases. Medical image analysis, 14(2), pp.227-241.

[10]. Bian, W., \& Tao, D. (2010). Biased discriminant euclidean embedding for content-based image retrieval. IEEE transactions on image processing, 19(2), pp.545-554.

[11]Yue, J., Li, Z., Liu, L. and Fu, Z., 2011. Content-based image retrieval using color and texture fused features. Mathematical and Computer Modelling, 54(3-4), pp.11211127.

[12]. Su, J. H., Huang, W. J., Philip, S. Y., \& Tseng, V. S. (2011). Efficient relevance feedback for content-based image retrieval by mining user navigation patterns. IEEE transactions on knowledge and data engineering, 23(3), 360372

[13]. Murala, S., Maheshwari, R. P., \&Balasubramanian, R. (2012). Local tetra patterns: a new feature descriptor for content-based image retrieval. IEEE Transactions on Image Processing, 21(5), pp.2874-2886.

[14]. Liu, G. H., \& Yang, J. Y. (2013). Content-based image retrieval using color difference histogram. Pattern recognition, 46(1), 188-198.

[15] Malik, F. and Baharudin, B., 2013. Analysis of distance metrics in content-based image retrieval using statistical quantized histogram texture features in the DCT domain. Journal of king saud university-computer and information sciences, 25(2), pp.207-218.

[16] Avinash N Bhute1, B. B. Meshram,'Content Based Image Indexing and Retrieval'International Journal of Graphics \& Image Processing |Vol 3|issue 4|Nov. 2013

[17]Munje, P.N. and KAPGATE, D., 2014. novel contentbased image retrieval techniques for medical database. International Journal, 3(6).

[18] Fu, R., Li, B., Gao, Y. and Wang, P., 2016, October. Content-based image retrieval based on CNN and SVM. In Computer and Communications (ICCC), 2016 2nd IEEE International Conference on (pp. 638-642). IEEE.

[19]. Alsmadi , "An efficient similarity measure for content based image retrieval using memetic algorithm", Egyptian Journal of Basic and Applied Sciences 4 (2017) pp.112-122. 